\section{ANTIBIOTIC DEVELOPMENT}

\section{No more ESKAPE}

Nature 561, 189-194 (2018)

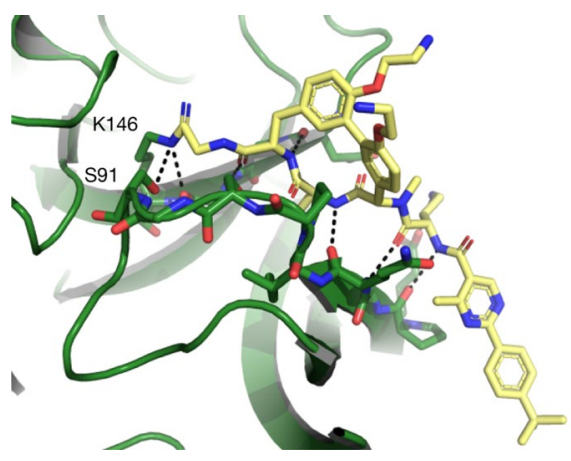

Credit: Nature

Arylomycins are macrocyclic lipopeptides that have shown promise as antibiotics against Gram-positive bacteria by targeting the active site of a signal peptidase (SPase) on the cell surface. However, these natural products have limited activity against Gram-negative bacteria owing to difficulty penetrating the outer-membrane permeability barrier. Smith et al. identified an arylomycin analog, G0775, which is 500-fold more potent than the original arylomycin against Gram-negative members of the ESKAPE pathogen group of bacteria, considered the most likely species to lead to untreatable multidrug-resistant (MDR) infections. G0775 remains potent against dozens of clinical isolates of ESKAPE pathogens, including an MDR isolate of Klebsiella pneumoniae that is resistant to 13 classes of antibiotics, and is efficacious in multiple murine infection models. The authors detected a low frequency of mutations in the Gram-negative SPase LepB that resulted in resistance to G0775, suggesting this protein as its target. Indeed, structural analysis showed that resistance mutations mapped to G0775-binding residues on LepB and revealed a covalent mechanism of inhibition. Finally, G0775 seems to enter Escherichia coli by a selfpromoted uptake that is enhanced by its positive charge, defining a unique mechanism of antibiotic entry and previously undrugged target in Gram-negative bacteria. $M B$

https://doi.org/10.1038/s41589-018-0156-7

\section{TRANSLATIONAL REGULATION}

A new spot for PKR

Mol. Cell 71, 1051-1063 (2018)

Recognition of viral dsRNA by PKR (protein kinase RNA-activated) causes its autophosphorylation and phosphorylation of eIF2 $\alpha$ (eukaryotic translation initiation factor 2 alpha), leading to global translational inhibition. Increasing evidence shows that PKR can also be phosphorylated during the cell cycle and under stress conditions without infection. To identify the endogenous dsRNAs that can activate PKR under these conditions, Kim et al. utilized a formaldehyde-mediated cross-linking and immunoprecipitation sequencing (fCLIPseq) approach to profile PKR-interacting RNAs in S- or M-phase-arrested HeLa cells. They found that the major class of PKR-interacting dsRNAs was derived from mitochondrial RNAs (mtRNAs). Decreasing the amount of mtRNAs by knocking down POLRMT, a mitochondrial RNA polymerase, reduced the level of phosphorylated PKR

\title{
PROTEIN QUALITY CONTROL
}

\section{Chaperoning partners}

Science https://doi.org/10.1126/science.aap8236 (2018)

The BTB protein domain functions as an interaction module, enabling self-association as a homodimer or interaction with non-BTB proteins within ubiquitin ligase or transcription factor complexes. While BTB domain proteins have the potential to form complexes with other BTB proteins, very few of these complexes, which are likely inactive, are detected in cells. To explore this discrepancy, Mena et al. generated artificial BTB heterodimers and observed interactions with the SCF E3 ligase adaptor FBXL17. In particular, FBXL17 interacted with specific residues in the BTB domain that were exposed in immature homodimers and BTB heterodimers but were buried in mature BTB homodimers. This interaction resulted in the ubiquitination and proteosomal degradation of aberrant BTB complexes while sparing mature BTB homodimers. MS analysis of endogenous BTB proteins in cells lacking FBXL17 revealed an increased abundance of aberrant complexes with other BTB proteins and an impaired ability to interact with their endogenous substrates. A similar phenotype was observed in Xenopus laevis embryos treated with a FBXL17 morpholino, with defects in neuronal and neuron crest differentiation. Overall, these findings reveal a unique quality control system that specifically prevents accumulation of protein complexes of aberrant composition, rather than detecting protein misfolding.

https://doi.org/10.1038/s41589-018-0157-6

(pPKR) and eIF2 $\alpha$. Redirecting PKR into the mitochondrial matrix only increased the level of pPKR, suggesting that mitochondrial export of pPKR is required for eIF $2 \alpha$ phosphorylation. During $M$ phase and under stress conditions, the upregulation of mtRNAs and/or cytosolic release of $m t R N A s$ results in increased mtRNA-PKR duplex formation and PKR activation. These results show that mtRNAs can induce PKR activation and provide new insight into the role of mtRNA in translational regulation.

YS

https://doi.org/10.1038/s41589-018-0159-4

\section{REACTIVE SULFUR SPECIES}

\section{Trading on thionoesters \\ J. Am. Chem. Soc. 140, 12574-12579 (2018)}

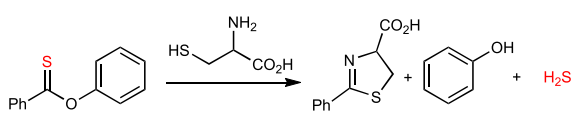

Credit: ACS

As a biological signaling molecule, hydrogen sulfide $\left(\mathrm{H}_{2} \mathrm{~S}\right)$ is involved in processes such as vasodilation and angiogenesis. Widely used $\mathrm{H}_{2} \mathrm{~S}$ sources such as sodium hydrosulfide release $\mathrm{H}_{2} \mathrm{~S}$ in a burst, unlike the more gradual release that occurs endogenously. This difference has led researchers to seek other approaches such as chemical tools that produce $\mathrm{H}_{2} \mathrm{~S}$ at slower, more physiologically relevant rates. Drawing inspiration from the native chemical ligation (NCL) reaction between a thioester and an N-terminal cysteine residue to generate an amide bond, Cerda et al. developed an analogous reaction using a thionoester (a structural isomer of thioesters) and cysteine, which produces $\mathrm{H}_{2} \mathrm{~S}$. The reaction between bis(phenyl) thionoester and free cysteine proceeds via nucleophilic addition and S-to-N acyl transfer, similarly to NCL, and robustly produces $\mathrm{H}_{2} \mathrm{~S}$, phenol and a stable dihydrothiazole. This reaction is selective for cysteine and homocysteine over other common biological thiols such as reduced glutathione, and proceeds under physiological conditions at rates comparable to those of copper-catalyzed azide-alkyne cycloadditions. Although the utility of the reaction has yet to be demonstrated in cells, it may be useful in the future development of tools for $\mathrm{H}_{2} \mathrm{~S}$ production or for ligation of reactive cysteine residues.

https://doi.org/10.1038/s41589-018-0158-5

Mirella Bucci, Caitlin Deane, Grant Miura and Yiyun Song 